\title{
Stable and Holomorphic Implementation of Complex Functions Using a Unit Circle-Based Transform
}

\author{
Binesh Thankappan (1) \\ Govt Model Engineering College-APJ Abdul Kalam Technological University, Ernakulam 682 021, India \\ Correspondence should be addressed to Binesh Thankappan; binesht@mec.ac.in
}

Received 20 September 2021; Revised 16 November 2021; Accepted 25 November 2021; Published 23 December 2021

Academic Editor: Gaetano Luciano

Copyright (c) 2021 Binesh Thankappan. This is an open access article distributed under the Creative Commons Attribution License, which permits unrestricted use, distribution, and reproduction in any medium, provided the original work is properly cited.

\begin{abstract}
A stable and holomorphic implementation of complex functions in $\mathbb{C}$ plane making use of a unit circle-based transform is presented in this paper. In this method, any complex number or function can be represented as an infinite series sum of progressive products of a base complex unit and its conjugate only, where both are defined inside the unit circle. With each term in the infinite progression lying inside the unit circle, the sum ultimately converges to the complex function under consideration. Since infinitely large number of terms are present in the progression, the first element of which may be deemed as the base unit of the given complex number, it is addressed as complex baselet so that the complex number or function is termed as the complex baselet transform. Using this approach, various fundamental operations applied on the original complex number in $\mathbb{C}$ are mapped to equivalent operations on the complex baselet inside the unit circle, and results are presented. This implementation has unique properties due to the fact that the constituent elements are all lying inside the unit circle. Out of numerous applications, two cases are presented: one of a stable implementation of an otherwise unstable system and the second case of functions not satisfying Cauchy-Riemann equations thereby not holomorphic in $\mathbb{C}$ plane, which are made complex differentiable using the proposed transform-based implementation. Various lemmas and theorems related to this approach are also included with proofs.
\end{abstract}

\section{Introduction}

Complex analysis has long been regarded as a powerful tool for solving problems in pure mathematics as well as in applied streams and engineering. Detailed studies in complex variables and analytic functions along with transforms and holomorphic analysis have been reported in [1-13]. Generalizations of Cauchy-Riemann equations, stability of polynomials, transfer functions, and analysis of complex spatial variables have been also been investigated extensively [14-18]. Significant results making use of complex analysis in applied mathematics and physics have also been reported with special relevance to electric circuits [19], quantum mechanics [20, 21], and quantum field theory $[22,23]$.

Theorem 1 introduces the basic concept of the proposed transform-based implementation of a complex number or an element of any complex function defined in $\mathbb{C}$ plane.
According to this theorem, any arbitrary function defined outside the unit circle can be mapped to a basic complex element defined inside the unit circle. Alternatively, this random complex function can be generated back, using its base element and the conjugate only, using an infinite progressive series that always converges.

Theorem 1. If $Z_{B}$ is a complex number defined outside the unit circle in $\mathbb{C}$ that can also be an element of an arbitrary complex function and can be represented in the form $Z_{B}=$ $\left(\left(x_{b}^{2}+x_{b}+y_{b}^{2}\right) /\left(1-x_{b}^{2}-y_{b}^{2}\right)\right)+\quad i\left(y_{b} /\left(1-x_{b}^{2}-y_{b}^{2}\right)\right)=$ $x+i y$, then $Z_{B}$ can be generated by the infinite progressive series $Z_{B}=\sum_{n=1, n \longrightarrow o d d}^{\infty-1} z_{b}^{n} e^{-i(n-1) \theta}+\sum_{n=2, n \longrightarrow \text { even }}^{\infty} z_{b}^{n} e^{-i n \theta}$, where the base unit $z_{b}=x_{b}+i y_{b}=r e^{i \theta}$ and all other components of the series being defined inside the unit circle, with $\left|z_{b}\right|=r<1$. Consequently, the entire $\mathbb{C}$ plane outside the unit circle can be mapped to inside the unit circle. 
Proof. The proof consists of three parts: Part 1, Part 2, and Lemma 1
Part 1. Defining an infinite series with sum $S$ and elements as progressive products of a base unit $z_{b}=x_{b}+i y_{b}=r e^{i \theta}$ and its complex conjugate $z_{b}^{*}=x_{b}-i y_{b}=r e^{-i \theta}$ as follows:

$$
\begin{aligned}
& S=\left(x_{b}+i y_{b}\right)+\left(x_{b}+i y_{b}\right)\left(x_{b}-i y_{b}\right)+\left(x_{b}+i y_{b}\right)\left(x_{b}-i y_{b}\right)\left(x_{b}+i y_{b}\right)+\cdots \text { infterms, } \\
& S=\left(x_{b}+i y_{b}\right)+g+g\left(x_{b}+i y_{b}\right)+g^{2}+\cdots \text { infterms, }
\end{aligned}
$$

where $g=r^{2}=x_{b}^{2}+y_{b}^{2}=\left|z_{b}\right|^{2}$, with $g<1$.

The sum converges as follows:

$$
\begin{aligned}
& S=\left(x_{b}+i y_{b}\right) \frac{1}{1-g}+\frac{g}{1-g}, \\
& S=\frac{x_{b}+i y_{b}+g}{1-g}=\frac{1}{1-x_{b}^{2}-y_{b}^{2}}\left(x_{b}^{2}+x_{b}+y_{b}^{2}+i y_{b}\right), \\
& S=\frac{\left(x_{b}^{2}+x_{b}+y_{b}^{2}\right)}{\left(1-x_{b}^{2}-y_{b}^{2}\right)}+i \frac{y_{b}}{\left(1-x_{b}^{2}-y_{b}^{2}\right)}=Z_{B} .
\end{aligned}
$$

Therefore, $Z_{B}$ can be represented as above, using a base unit $z_{b}$ inside the unit circle, provided $z_{b}$ exists.

Part 2. Proof for existence of base unit inside the unit circle for an arbitrary complex number or function in $\mathbb{C}$.

From Part 1, any arbitrary complex number outside the unit circle can be expressed in the form as follows:

$$
x+i y=Z_{B}=\frac{\left(x_{b}^{2}+x_{b}+y_{b}^{2}\right)}{\left(1-x_{b}^{2}-y_{b}^{2}\right)}+i \frac{y_{b}}{\left(1-x_{b}^{2}-y_{b}^{2}\right)} .
$$

The real part is expressed as follows:

$$
x=\frac{\left(x_{b}+x_{b}^{2}+y_{b}^{2}\right)}{\left(1-x_{b}^{2}-y_{b}^{2}\right)}
$$

where $\left(x_{b}^{2}+y_{b}^{2}\right)<1$.

The imaginary part is expressed as follows:

$$
y=\frac{y_{b}}{\left(1-x_{b}^{2}-y_{b}^{2}\right)} \text {. }
$$

The solutions for $x_{b}$ and $y_{b}$ can be extracted from the following set of equations and conditions of inequalities:

$$
\begin{aligned}
& x_{b}=\frac{\left.1 \pm \sqrt{1-4(x+1)\left(\left(\left(-1 \pm \sqrt{1+4 y\left(y-x_{b}^{2}\right)} / 2 y\right)\right)^{2}(x+1)-x\right.}\right)}{2(x+1)} \\
& y_{b}=\frac{-1 \pm \sqrt{1+4 y\left(y-\left(\left(1 \pm \sqrt{1-4(x-1)(x+1) y_{b}^{2}} / 2(x-1)\right)\right)^{2}\right.}}{2 y} .
\end{aligned}
$$

The additional conditions of inequalities are as follows:

$$
\begin{aligned}
& y_{b}^{2}<4 x-\frac{x(x-1)}{(x+1)^{2}}, \\
& x_{b}^{2}<-4 x+\frac{3 x+1}{(x+1)^{2}} .
\end{aligned}
$$

Therefore, the base unit $x_{b}+i y_{b}$ of the complex number or function $Z_{B}$ in $\mathbb{C}$ exists inside the unit circle.

Lemma 1. The integer $\infty$ defined in the complex plane $\mathbb{C}$ for the vanishing progression is even, and therefore the integer $\infty-1$ is odd.

Proof. From Part 1 of proof, the sum to infinite terms is

$$
S=Z_{B}=\left(x_{b}+i y_{b}\right)+g+g\left(x_{b}+i y_{b}\right)+g^{2}+g^{2}\left(x_{b}+i y_{b}\right)+\ldots \text { infterms }
$$

Analyzing the lemma, with $x_{b}<1, y_{b}<1$, and also $g<1$, the series is converging, and assuming at $\infty$ (infinity), an odd term vanishes to zero. If that happens, the next even term and all subsequent terms will be zero, and therefore, for the series, $\infty$ has to be defined at that odd integer $(n)$ term under consideration. Alternatively, for the series, if this odd 
integer $n$ is defined as $\infty$, then the term $g^{n}\left(x_{b}+i y_{b}\right)$ has to vanish. This happens only when $g^{n}$ is vanished to zero because $x_{b}$ and $y_{b}$ are finite within the unit circle. This implies that if an odd integer is defined as $\infty$ for the series, $g^{n}$ in that term would have already vanished. However, $g^{n}$ constitutes the even term just before the $n^{\text {th }}$ term, i.e., the first term that vanishes-which would be the last term of the progression-cannot be an odd integer term in the series, and therefore, the integer $\infty$ defined in the complex plane $\mathbb{C}$ for the vanishing progressive series is even. Consequently, the integer $\infty-1$ will be odd.

Using Part 1, Part 2, and Lemma 1, the transform can be represented as follows: $Z_{B}=\sum_{n=1, n \longrightarrow \text { odd }}^{\infty-1} z_{b}^{n} e^{-i(n-1) \theta_{+}}$ $\sum_{n=2, n \longrightarrow \text { even }}^{\infty} z_{b}^{n} e^{-i n \theta}$, where the base unit $z_{b}=x_{b}+i y_{b}=r e^{i \theta}$ and all other components of the series being defined inside the unit circle, with the condition $\left|z_{b}\right|<1$. So, the entire $\mathbb{C}$ plane outside the unit circle can be mapped to inside the unit circle.

Thus, the theorem is proved.

\section{Terminologies: Complex Baselet and Complex Baselet Transform}

Because an infinite progressive series representation of a complex number or function is used in the approach, the first element of which may be deemed as the base unit of the given complex number, the terminology complex baselet [3] denoted by $z_{b}$ is used in the paper and the complex number or function is termed as the complex baselet transform [3] of $z_{b}$, denoted by $Z_{B}$.

The complex baselet transform of $z_{b}$ is formally defined from Theorem 1 as follows: $Z_{B}=\sum_{n=1, n \longrightarrow \text { odd }}^{\infty-1} z_{b}^{n} e^{-i(n-1) \theta_{+}}$ $\sum_{n=2, n \rightarrow \text { even }}^{\infty} z_{b}^{n} e^{-i n \theta}$, where $z_{b}=x_{b}+i y_{b}=r e^{i \theta}$ and all other elements of the series being defined inside the unit circle, with $\left|z_{b}\right|<1$. The given complex number $Z_{B}$ outside the unit circle becomes the complex baselet transform of $z_{b}$. Additionally, the inverse complex baselet transform can be defined recursively as follows: $z_{b}=\left(1 / \sum_{n=1, n \longrightarrow \text { odd }}^{\infty-1}\right.$ $\left.\left(1 / Z_{B}\right) r^{n-1}+\sum_{n=2, n \rightarrow \text { even }}^{\infty}\left(r^{n-1} e^{-i \theta} / Z_{B}\right)\right)$ even though estimation of $z_{b}$ can be carried out using Part 2 of proof in the aforementioned theorem. However, this recursive relation is useful for further analysis.

Lemma 2. If $z_{b}=x_{b}+i y_{b}=r e^{i \theta}$, defined inside the unit circle is the complex baselet of $Z_{B}=x+i y$, in $\mathbb{C}$ outside the unit circle, then $\lim _{(n+1) \longrightarrow \infty}\left(z_{b}^{*} z_{b}^{n} e^{-i(n-1) \theta}\right)=\lim _{n \longrightarrow \infty}$ $\left(z_{b}^{n} e^{-i n \theta}\right)=0$, where $z_{b}^{*}$ represents the complex conjugate of $z_{b}$.
Proof. Proof of Lemma 2 follows from Theorem 1 and Lemma 1

\section{Theorems on Complex Baselet Transform}

Theorem 2. Conjugate operation of a complex number in $\mathbb{C}$ plane is transformed as is to the complex baselet inside the unit circle, i.e., when $Z_{B}=x+i y$ becomes $Z_{B}^{*}=x-i y$ in $\mathbb{C}$ plane, the complex baselet gets transformed to $z_{b}^{*}=x_{b}-i y_{b}=$ re ${ }^{-i \theta}$ from $z_{b}=x_{b}+i y_{b}=r e^{i \theta}$ inside the unit circle.

Proof. Assume as proposed in the theorem, $z_{b}^{*}=x_{b}-i y_{b}=$ $r e^{-i \theta}$ is the complex baselet of $Z_{B}^{*}=x-i y$. Next, the basic generation series becomes

$$
\begin{aligned}
S= & \left(x_{b}-i y_{b}\right)+\left(x_{b}-i y_{b}\right)\left(x_{b}+i y_{b}\right)+\left(x_{b}-i y_{b}\right) \\
& \left(x_{b}+i y_{b}\right)\left(x_{b}-i y_{b}\right)+\cdots \text { infterms },
\end{aligned}
$$

$S=\left(x_{b}-i y_{b}\right)(1 / 1-g)+(g / 1-g)$, where $g=r^{2}=x_{b}^{2}+$ $y_{b}^{2}=\left|z_{b}\right|^{2}$ with $g<1$,

$$
S=\frac{x_{b}-i y_{b}+g}{1-g}=\frac{1}{1-x_{b}^{2}-y_{b}^{2}}\left(x_{b}^{2}+x_{b}+y_{b}^{2}-i y_{b}\right) \text {, }
$$

and $S=\left(\left(x_{b}^{2}+x_{b}+y_{b}^{2}\right) /\left(1-x_{b}^{2}-y_{b}^{2}\right)\right)-i \quad\left(y_{b} /\left(1-x_{b}^{2}-\right.\right.$ $\left.\left.y_{b}^{2}\right)\right)=Z_{B}^{*}=x-i y$ in $\mathbb{C}$.

Therefore, conjugate operation on $Z_{B}$ in $\mathbb{C}$ is transformed as is to $z_{b}$ inside the unit circle.

Theorem 3. Let $Z_{B}$ be a complex number defined outside the unit circle in $\mathbb{C}$, which is the complex baselet transform of $z_{b}$ defined inside the unit circle, with the scalar $r=\left|z_{b}\right|$ and $r<1$, then the operation on $Z_{B}, \quad F\left(Z_{B}\right)=\left(1 / \sum_{n=1, n \longrightarrow \text { odd }}^{\infty}\right.$ $\sum_{k=1}^{N} e^{(-1)^{k} i \theta}\left(1 / Z_{B}\right) r^{n-1}+\sum_{n=2, n \longrightarrow \text { even }}^{\infty-1} \sum_{k=1}^{N} e^{(-1)^{k} i \theta} \quad\left(1 / Z_{B}\right)$ $\left.r^{n-1} e^{-i \theta}\right)$ carried out in $\mathbb{C}$ produces a transformation $z_{t r}$ on the complex baselet $z_{b}$ of $Z_{B}$ inside the unit circle, as the solution of $Z_{B}=N \cos \theta \sum_{n=1, n \longrightarrow o d d}^{\infty-1}\left(z_{t r} \sec \theta / N\right)^{n}$ $e^{-i(n-1) \theta}+\sum_{n=2, n \longrightarrow \text { even }}^{\infty}\left(z_{t r} \sec \theta / N\right)^{n} e^{-i n \theta} \quad$ with $\left|z_{t r} \sec \theta / N\right|<1$, for $N$ even and

$$
\begin{aligned}
Z_{B}= & \frac{1}{\left((N-1) \cos \theta+e^{-i \theta}\right)} \sum_{n=1, n \longrightarrow \text { odd }}^{\infty-1}\left(\frac{z_{t r} \sec \theta}{N}\right)^{n} e^{-i(n-1) \theta} \\
& +\sum_{n=2, n \longrightarrow \text { even }}^{\infty}\left(\frac{z_{t r} \sec \theta}{N}\right)^{n} e^{-i n \theta},
\end{aligned}
$$

with $\left|\left(z_{t r} /(N-1) \cos \theta+e^{-i \theta}\right)\right|<1$, for $N$ odd.

Proof. Let the given operation on $Z_{B}$ produce a transformation $T\left(z_{b}\right)$ on $z_{b}$ as follows:

$$
T\left(z_{b}\right)=\frac{1}{\sum_{k=1}^{N} e^{(-1)^{k} i \theta}} \frac{1}{\sum_{n=1, n \longrightarrow \text { odd }}^{\infty}\left(1 / Z_{B}\right) r^{n-1}+\sum_{n=2, n \longrightarrow \text { even }}^{\infty-1}\left(1 / Z_{B}\right) r^{n-1} e^{-i \theta}} .
$$


This can be expressed as follows:

$$
T\left(z_{b}\right)=\frac{1}{\left(e^{-i \theta}+e^{i \theta}+e^{-i \theta}+\cdots N \text { terms }\right) \sum_{n=1, n \longrightarrow \text { odd }}^{\infty}\left(1 / Z_{B}\right) r^{n-1}+\sum_{n=2, n \longrightarrow \text { even }}^{\infty-1}\left(1 / Z_{B}\right) r^{n-1} e^{-i \theta}}
$$

For $N$ even,

$$
T\left(z_{b}\right)=\frac{1}{N \cos \theta} \frac{1}{\sum_{n=1, n \longrightarrow \text { odd }}^{\infty}\left(1 / Z_{B}\right) r^{n-1}+\sum_{n=2, n \longrightarrow \text { even }}^{\infty-1}\left(1 / Z_{B}\right) r^{n-1} e^{-i \theta}},
$$

i.e., $T\left(z_{b}\right)=(1 / N) z_{b} \sec \theta$, for $N$ even, with the condition

For $N$ odd, $\left|\left(z_{b} \sec \theta / N\right)\right|<1$ for the complex baselet to be inside the unit circle.

$$
T\left(z_{b}\right)=\frac{1}{\left((N-1) \cos \theta+e^{-i \theta}\right)} \frac{1}{\sum_{n=1, n \longrightarrow \text { odd }}^{\infty}\left(1 / Z_{B}\right) r^{n-1}+\sum_{n=2, n \longrightarrow \text { even }}^{\infty-1}\left(1 / Z_{B}\right) r^{n-1} e^{-i \theta}}
$$

and $T\left(z_{b}\right)=\left(z_{b} /\left((N-1) \cos \theta+e^{-i \theta}\right)\right)$, with the condition $\left|\left(z_{b} /\left((N-1) \cos \theta+e^{-i \theta}\right)\right)\right|<1$ for the complex baselet to be

Therefore, the operation produces a new element in $\mathbb{C}$ : inside the unit circle.

$$
Z_{B \text { trans }}=\frac{Z_{B}}{N \cos \theta}=\sum_{n=1, n \longrightarrow \text { odd }}^{\infty-1}\left(\frac{z_{t r} \sec \theta}{N}\right)^{n} e^{-i(n-1) \theta}+\sum_{n=2, n}^{\infty}\left(\frac{z_{t r} \sec \theta}{N}\right)^{n} e^{-i n \theta}
$$

with $\left|\left(z_{t r} \sec \theta / N\right)\right|<1$ for $N$ even.

Alternatively, $z_{t r}$ is the solution of

$$
Z_{B}=N \cos \theta \sum_{n=1, n \longrightarrow \text { odd }}^{\infty-1}\left(\frac{z_{t r} \sec \theta}{N}\right)^{n} e^{-i(n-1) \theta}+\sum_{n=2, n \longrightarrow \text { even }}^{\infty}\left(\frac{z_{t r} \sec \theta}{N}\right)^{n} e^{-i n \theta}
$$

with $\left|\left(z_{t r} \sec \theta / N\right)\right|<1$, for $N$ even.

Similarly, $z_{t r}$ is the solution of

$$
Z_{B}=\frac{1}{\left((N-1) \cos \theta+e^{-i \theta}\right)} \sum_{n=1, n \longrightarrow \text { odd }}^{\infty-1}\left(\frac{z_{t r}}{\left((N-1) \cos \theta+e^{-i \theta}\right)}\right)^{n} e^{-i(n-1) \theta}+\sum_{n=2, n \longrightarrow \text { even }}^{\infty}\left(\frac{z_{t r}}{\left((N-1) \cos \theta+e^{-i \theta}\right)}\right)^{n} e^{-i n \theta}
$$

with the condition $\left|\left(z_{t r} /(N-1) \cos \theta+e^{-i \theta}\right)\right|<1$, for $N$ odd.

Thus, the theorem is proved.
Corollary 1. Let a multiplication operation $R=Z_{2} Z_{B}=r_{2} e^{i \phi} Z_{B}$ be defined in $\mathbb{C}$, for $Z_{B}$, a complex 
number defined outside the unit circle, which is the complex baselet transform of $z_{b}$ defined inside the unit circle, then the operation transforms the original complex baselet $z_{b}$ of $Z_{B}$ to $z_{t r} r_{2} e^{i \phi}$, while $z_{t r}$ is the solution of

$$
\begin{aligned}
R= & \sum_{n=1, n \longrightarrow \text { odd }}^{\infty-1}\left(z_{t r} r_{2}\right)^{n} e^{-i((n-1)(\theta+\phi))} \\
& +\sum_{n=2, n}^{\infty}\left(z_{t r}\right)^{n} e^{-i n(\theta+\phi)},
\end{aligned}
$$

with the condition $\left|z_{t r} r_{2}\right|<1$ and $\arg \left(z_{t r}\right)=\theta+\phi$.

Proof. From Theorem 3, the operation becomes a transformation $T\left(z_{b}\right)$ on $z_{b}$ as

$$
T\left(z_{b}\right)=\frac{r_{2} e^{i \phi}}{\sum_{n=1, n \longrightarrow \text { odd }}^{\infty}\left(1 / Z_{B}\right) r^{n-1}+\sum_{n=2, n \longrightarrow \text { even }}^{\infty-1}\left(r^{n-1} e^{-i \theta} / Z_{B}\right)} .
$$

Next, $z_{b}$ is modified to a complex quantity inside the unit circle $z_{t r} r_{2} e^{i \phi}$, with $z_{t r}$ being the solution of

$$
\begin{aligned}
R= & \sum_{n=1, n \longrightarrow \text { odd }}^{\infty-1}\left(z_{t r} r_{2}\right)^{n} e^{-i((n-1)(\theta+\phi))} \\
& +\sum_{n=2, n}^{\infty}\left(z_{t r}\right)^{n} e^{-i n(\theta+\phi)},
\end{aligned}
$$

along with the condition $\left|z_{t r} r_{2}\right|<1$ and $\arg \left(z_{t r}\right)=\theta+\phi$.

Corollary 2. Let a division operation $R=\left(Z_{B} / Z_{2}\right)=$ $\left(Z_{B} / r_{2} e^{i \phi}\right)$ be defined in $\mathbb{C}$, on $Z_{B}$, a complex number defined outside the unit circle, which is the complex baselet transform of $z_{b}$ defined inside the unit circle, then the operation transforms the original complex baselet $z_{b}$ of $Z_{B}$ to $\left(z_{t r} / r_{2} e^{i \phi}\right)$, while $z_{\text {tr }}$ is the solution of

$$
\begin{aligned}
R= & \sum_{n=1, n \longrightarrow \text { odd }}^{\infty-1}\left(\frac{z_{t r}}{r_{2}}\right)^{n} e^{-i((n-1)(\theta-\phi))} \\
& +\sum_{n=2, n \longrightarrow \text { even }}^{\infty}\left(\frac{z_{t r}}{r_{2}}\right)^{n} e^{-i n(\theta-\phi)},
\end{aligned}
$$

with the condition $\left|\left(z_{t r} / r_{2}\right)\right|<1$ and $\arg \left(z_{t r}\right)=\theta-\phi$.

Proof. The proof follows from Corollary 1, with $z_{b}$ modified to $\left(z_{t r} / r_{2} e^{i \phi}\right)$, where $z_{t r}$ is the solution of

$$
R=\sum_{n=1, n \longrightarrow \text { odd }}^{\infty-1}\left(\frac{z_{t r}}{r_{2}}\right)^{n} e^{-i((n-1)(\theta-\phi))}+\sum_{n=2, n \longrightarrow \text { even }}^{\infty}\left(\frac{z_{t r}}{r_{2}}\right)^{n} e^{-i(\theta-\phi)},
$$

with the condition $\left|\left(z_{t r} / r_{2}\right)\right|<1$ and $\arg \left(z_{t r}\right)=\theta-\phi$.

Theorem 4. If $Z_{B 1}$ and $Z_{B 2}$ are two complex numbers or functions defined outside the unit circle in $\mathbb{C}$, with their respective complex baselets $z_{b 1}$ and $z_{b 2}$ defined inside the unit circle, the summation operation of $z_{b 1}$ and $z_{b 2}$ produces a function $z_{s}=F\left(Z_{B 1}, Z_{B 2}\right)$ such that

$$
z_{s}=\left(Z_{B 1}\left(1-\left|z_{b 1}\right|^{2}\right)-\left|z_{b 1}\right|^{2}\right)+\left(Z_{B 2}\left(1-\left|z_{b 2}\right|^{2}\right)-\left|z_{b 2}\right|^{2}\right) .
$$

Proof. Let two complex numbers or functions in $\mathbb{C}$ be $Z_{B 1}$ and $Z_{B 2}$, and their respective complex baselets be $z_{b 1}$ and $z_{b 2}$, and the sum of complex baselets be $z_{s}$.

From Theorem 1, let for any complex number or function $Z_{B}$ in $\mathbb{C}$,

$$
S=\left(x_{b}+i y_{b}\right) \frac{1}{1-g}+\frac{g}{1-g} .
$$

Substituting and simplifying, the following relation can be obtained:

$$
z_{b}=\left(1-\left|z_{b}\right|^{2}\right)\left[Z_{B}-\frac{\left|z_{b}\right|^{2}}{\left(1-\left|z_{b}\right|^{2}\right)}\right]
$$

with the condition $\left|z_{b}\right|<1$.

For $Z_{B 1}$,

$$
z_{b 1}=\left(1-\left|z_{b 1}\right|^{2}\right)\left[Z_{B 1}-\frac{\left|z_{b 1}\right|^{2}}{\left(1-\left|z_{b 1}\right|^{2}\right)}\right] .
$$

For $Z_{B 2}$,

$$
z_{b 2}=\left(1-\left|z_{b 2}\right|^{2}\right)\left[Z_{B 2}-\frac{\left|z_{b 2}\right|^{2}}{\left(1-\left|z_{b 2}\right|^{2}\right)}\right] .
$$

The sum of two complex baselets which are individually defined inside the unit circle is related to $Z_{B 1}$ and $Z_{B 2}$ as follows:

$$
\begin{aligned}
z_{s}= & \left(1-\left|z_{b 1}\right|^{2}\right)\left[Z_{B 1}-\frac{\left|z_{b 1}\right|^{2}}{\left(1-\left|z_{b 1}\right|^{2}\right)}\right] \\
& +\left(1-\left|z_{b 2}\right|^{2}\right)\left[Z_{B 2}-\frac{\left|z_{b 2}\right|^{2}}{\left(1-\left|z_{b 2}\right|^{2}\right)}\right] \\
z_{s}= & \left(Z_{B 1}\left(1-\left|z_{b 1}\right|^{2}\right)-\left|z_{b 1}\right|^{2}\right)+\left(Z_{B 2}\left(1-\left|z_{b 2}\right|^{2}\right)-\left|z_{b 2}\right|^{2}\right) .
\end{aligned}
$$

Corollary 3. If $Z_{B 1}$ and $Z_{B 2}$ are two complex numbers or functions defined outside the unit circle in $\mathbb{C}$, with their respective complex baselets $z_{b 1}$ and $z_{b 2}$ defined inside the unit circle, then the operation in $\mathbb{C}: Z_{\text {sum }}=Z_{B 1}+Z_{B 2}$ corresponds to a complex baselet $z_{s}$ inside the unit circle as follows:

$$
z_{s}=\left(1-\left|z_{s}\right|^{2}\right)\left[\left(Z_{B 1}+Z_{B 2}\right)-\frac{\left|z_{s}\right|^{2}}{\left(1-\left|z_{s}\right|^{2}\right)}\right],
$$

with $\left|z_{s}\right|<1$. 
Proof. The inverse complex baselet transform equation is derived as follows:

$$
z_{b}=\left(1-\left|z_{b}\right|^{2}\right)\left[Z_{B}-\frac{\left|z_{b}\right|^{2}}{\left(1-\left|z_{b}\right|^{2}\right)}\right]
$$

Let the complex baselet transform be $z_{s}$, for $Z_{\text {sum }}=Z_{B 1}+Z_{B 2}$. Therefore, the relation modifies to the following:

$$
z_{s}=\left(1-\left|z_{s}\right|^{2}\right)\left[\left(Z_{B 1}+Z_{B 2}\right)-\frac{\left|z_{s}\right|^{2}}{\left(1-\left|z_{s}\right|^{2}\right)}\right],
$$

with $\left|z_{s}\right|<1$.

Thus, the theorem is proved.

Corollary 4. If $Z_{B 1}$ and $Z_{B 2}$ are two complex numbers or functions defined outside the unit circle in $\mathbb{C}$, with their respective complex baselets $z_{b 1}$ and $z_{b 2}$ defined inside the unit circle, then the operation outside unit circle in $\mathbb{C}: Z_{\text {diff }}=$ $Z_{B 1}-Z_{B 2}$ corresponds to a complex baselet $z_{d}$ inside the unit circle as follows:

$$
z_{d}=\left(1-\left|z_{d}\right|^{2}\right)\left[\left(Z_{B 1}-Z_{B 2}\right)-\frac{\left|z_{d}\right|^{2}}{\left(1-\left|z_{d}\right|^{2}\right)}\right],
$$

with $\left|z_{d}\right|<1$.

Proof. The inverse complex baselet transform equation is derived as follows:

$$
z_{b}=\left(1-\left|z_{b}\right|^{2}\right)\left[Z_{B}-\frac{\left|z_{b}\right|^{2}}{\left(1-\left|z_{b}\right|^{2}\right)}\right] .
$$

For $Z_{\text {diff }}=Z_{B 1}-Z_{B 2}$, let the complex baselet transform be $z_{d}$. Then, the relation modifies to the following:

$$
z_{d}=\left(1-\left|z_{d}\right|^{2}\right)\left[\left(Z_{B 1}-Z_{B 2}\right)-\frac{\left|z_{d}\right|^{2}}{\left(1-\left|z_{d}\right|^{2}\right)}\right],
$$

with $\left|z_{d}\right|<1$.

Thus, the theorem is proved.

Theorem 5. Unstable systems whose poles of the transfer function $H(Z)$ lie outside the unit circle in $Z$ domain can be implemented in a stable configuration, with no poles outside the unit circle, making use of complex baselet transform.

Proof. A system whose transfer function defined in the $Z$ domain is stable if all its poles lie within the unit circle in the $Z$ plane. Let the transfer function of the unstable system be expressed in the form: $H(Z)=((A Z+B)(C Z+D)$ $\left.\ldots /\left(Z-p_{1}\right)\left(Z-p_{2}\right) \ldots\right)$, where the poles $p_{1}, p_{2}, \ldots$ are complex quantities lying outside the unit circle. Transform $H(Z)=\left(r_{n r} e^{i \alpha} / r_{d r 1} e^{i \beta_{1}} r_{d r 2} e^{i} \quad \beta_{2} \ldots\right)=\left(r_{n r} / r_{d r}\right)$ $e^{i\left(\alpha-\beta_{1}-\beta_{2}-\ldots\right)}=\left(r_{n r} / r_{d r}\right) e^{i(\alpha-\beta)}$, where $r_{d r}=r_{d r 1}, r_{d r 2}, \ldots$ and $\beta=\left(\beta_{1}+\beta_{2}+\ldots\right)$. Let $z_{b}=r e^{i \theta}$ be the complex baselet of the numerator of the transfer function, defined inside the unit circle. Due to the presence of denominator whereby poles are present outside the unit circle for this function, the new transformed complex baselet denoted by $\mathrm{z}_{t r}$ will be the solution of the equation with converging RHS due to a necessary condition, expressed as follows. Therefore,

$$
\begin{aligned}
H(Z)= & \sum_{n=1, n \longrightarrow \text { odd }}^{\infty-1}\left(\frac{z_{t r}}{r_{d r}}\right)^{n} e^{-i((n-1)(\theta-\beta))} \\
& +\sum_{n=2, n}^{\infty}\left(\frac{z_{t r}}{r_{d r}}\right)^{n} e^{-i(\theta-\beta)}
\end{aligned}
$$

with the condition $\left|\left(z_{t r} / r_{d r}\right)\right|<1$.

When implemented inside the unit circle as in equation (36), the transfer function $H(Z)$ does not have any poles outside the unit circle and is stable. Therefore, those unstable systems whose poles lie outside the unit circle in $Z$ domain can be implemented in a stable configuration, using complex baselet transform.

Theorem 6. A counter clockwise rotation defined inside the unit circle on the complex baselet $f=z_{b} e^{i \phi}$ satisfies the Cauchy-Riemann equations with the partial derivatives defined with respect to $x_{b}$ and $y_{b}$ and hence differentiable with respect to $z_{b}$ and holomorphic inside the unit circle. The derivative of $f$ becomes the same counter clockwise rotation of the derivative of $z_{b}$, which becomes $e^{i \phi}$.

Proof. Let a counter clockwise rotation be defined on the complex baselet $z_{b}$ inside the unit circle as follows:

$$
f=z_{b} e^{i \phi} \text {. }
$$

Next, $f=\left(x_{b}+i y_{b}\right)(\cos \phi+i \sin \phi)$, lying inside the unit circle can be expressed as follows:

$$
f=\left(x_{b} \cos \phi-y_{b} \sin \phi\right)+i\left(x_{b} \sin \phi+y_{b} \cos \phi\right) \text {. }
$$

Applying Cauchy-Riemann equations inside the unit circle,

$$
\begin{gathered}
\frac{\partial U}{\partial x_{b}}=\cos \phi, \\
\frac{\partial V}{\partial y_{b}}=\cos \phi, \\
\frac{\partial U}{\partial y_{b}}=-\sin \phi, \\
-\frac{\partial V}{\partial x_{b}}=-\sin \phi .
\end{gathered}
$$

Therefore,

$$
\frac{\mathrm{d} f}{\mathrm{~d} z_{b}}=\frac{\partial U}{\partial x_{b}}+i \frac{\partial V}{\partial x_{b}}=\cos \phi+i \sin \phi=e^{i \phi} .
$$

From equations

and 
Theorem 7. The first derivative of a counter clockwise rotation defined inside the unit circle on the $n^{\text {th }}$ power of the complex baselet, $f=z_{b}^{n} e^{i m \phi}$, where $n$ and $m$ are integers, becomes the same counter clockwise rotation of the derivative of $z_{b}^{n}$, which becomes $e^{i m \phi} n z_{b}^{n-1}$.

Proof. For $f=z_{b} e^{i m \phi}$, using de Moivre's theorem and Theorem 6 as follows:

$$
\frac{\mathrm{d} f}{\mathrm{~d} z_{b}}=e^{i m \phi} \frac{\mathrm{d} z_{b}}{\mathrm{~d} z_{b}}=e^{i m \phi} .
$$

For $f=z_{b}^{n} e^{i m \phi}$,

$$
\frac{\mathrm{d} f}{\mathrm{~d} z_{b}}=\frac{\mathrm{d}\left(z_{b} e^{i m \phi} z_{b}^{n-1}\right)}{\mathrm{d} z_{b}} .
$$

Differentiating using product rule and also using equation (41),

$$
\begin{aligned}
& \frac{\mathrm{d} f}{\mathrm{~d} z_{b}}=z_{b} e^{i m \phi} \frac{\mathrm{d} z_{b}^{n-1}}{\mathrm{~d} z_{b}}+z_{b}^{n-1} e^{i m \phi}, \\
& \frac{\mathrm{d} f}{\mathrm{~d} z_{b}}=z_{b} e^{i m \phi}(n-1) z_{b}^{n-2}+z_{b}^{n-1} e^{i m \phi} .
\end{aligned}
$$

Therefore, $\left(\mathrm{d} f / \mathrm{d} z_{b}\right)=e^{i m \phi} n z_{b}^{n-1}=e^{i m \phi}\left(\mathrm{d} z_{b}^{n} / \mathrm{d} z_{b}\right)$.

Theorem 8. A clockwise rotation defined inside the unit circle on the complex baselet as $f=z_{b} e^{-i \phi}$ satisfies the Cauchy-Riemann equations, with the partial derivatives defined with respect to $x_{b}$ and $y_{b}$ and thus differentiable with respect to $z_{b}$ and holomorphic inside the unit circle. The derivative of $f$ becomes a clockwise rotation of the derivative of $z_{b}$ by the same angle, which will be $e^{-i \phi}$.

Proof. Let a clockwise rotation be defined on the complex baselet $z_{b}$ as follows:

$$
f=z_{b} e^{-i \phi} .
$$

Then, $f=\left(x_{b}+i y_{b}\right)(\cos \phi-i \sin \phi)$, inside the unit circle.

$$
f=\left(x_{b} \cos \phi+y_{b} \sin \phi\right)+i\left(y_{b} \cos \phi-x_{b} \sin \phi\right) .
$$

For verifying the function to be holomorphic, applying Cauchy-Riemann equations inside the unit circle,

$$
\begin{aligned}
\frac{\partial U}{\partial x_{b}} & =\cos \phi, \\
\frac{\partial V}{\partial y_{b}} & =\cos \phi, \\
\frac{\partial U}{\partial y_{b}} & =\sin \phi, \\
-\frac{\partial V}{\partial x_{b}} & =\sin \phi .
\end{aligned}
$$

Therefore,

$$
\frac{\mathrm{d} f}{\mathrm{~d} z_{b}}=\frac{\partial U}{\partial x_{b}}+i \frac{\partial V}{\partial x_{b}}=\cos \phi-i \sin \phi=e^{-i \phi} .
$$

$$
\begin{aligned}
& \text { From equations (44) and } \quad(47), \quad\left(\mathrm{d} f / \mathrm{d} z_{b}\right)= \\
& e^{-i \phi}\left(\mathrm{d} z_{b} / \mathrm{d} z_{b}\right)=e^{-i \phi} .
\end{aligned}
$$

Theorem 9. The first derivative of a clockwise rotation defined inside the unit circle on the $n^{\text {th }}$ power of the complex baselet, $f=z_{b}^{n} e^{-i m \phi}$, where $n$ and $m$ are integers, will be equivalent to a clockwise rotation of the derivative of $z_{b}^{n}$, by the same angle, which becomes $e^{-i m \phi} n z_{b}^{n-1}$.

Proof. For $f=z_{b} e^{-i m \phi}$, using de Moivre's theorem and Theorem 8,

$$
\frac{\mathrm{d} f}{\mathrm{~d} z_{b}}=e^{-i m \phi} \frac{\mathrm{d} z_{b}}{\mathrm{~d} z_{b}}=e^{-i m \phi}
$$

For $f=z_{b}^{n} e^{-i m \phi}$,

$$
\frac{\mathrm{d} f}{\mathrm{~d} z_{b}}=\frac{\mathrm{d}\left(z_{b} e^{-i m \phi} z_{b}^{n-1}\right)}{\mathrm{d} z_{b}} .
$$

Differentiating using product rule and also using Theorem 8 ,

$$
\begin{aligned}
& \frac{\mathrm{d} f}{\mathrm{~d} z_{b}}=z_{b} e^{-i m \phi} \frac{\mathrm{d} z_{b}^{n-1}}{\mathrm{~d} z_{b}}+z_{b}^{n-1} e^{-i m \phi}, \\
& \frac{\mathrm{d} f}{\mathrm{~d} z_{b}}=z_{b} e^{-i m \phi}(n-1) z_{b}^{n-2}+z_{b}^{n-1} e^{-i m \phi} .
\end{aligned}
$$

Therefore, $\left(\mathrm{d} f / \mathrm{d} z_{b}\right)=e^{-i m \phi} n z_{b}^{n-1}=e^{-i m \phi}\left(\mathrm{d} z_{b}^{n} / \mathrm{d} z_{b}\right)$.

Theorem 10. The nonanalytic function $f(Z)=Z_{B}^{*}$ defined in $\mathbb{C}$ outside the unit circle and not satisfying the Cauchy-Riemann equations which constitute a necessary and sufficient condition for it to be complex differentiable in $\mathbb{C}$, when implemented using the complex baselet, can be made complex differentiable inside the unit circle. Consequently, any complex differential operation on $Z_{B}^{*}$ in $\mathbb{C}$, which does not exist, is made equivalent to a differential operation on the complex baselet-based implementation inside the unit circle, which always converges and therefore exists.

Proof. First, complex derivative of $Z_{B}$ outside the unit circle is computed as follows:

$$
Z_{B}=\sum_{n=1, n \longrightarrow \text { odd }}^{\infty-1} z_{b}^{n} e^{-i(n-1) \theta}+\sum_{n=2, n \longrightarrow \text { even }}^{\infty} z_{b}^{n} e^{-i n \theta} .
$$

Furthermore, 


$$
\begin{aligned}
\frac{\mathrm{d} Z_{B}}{\mathrm{~d} Z}=\frac{\mathrm{d} Z_{B}}{\mathrm{~d} z_{b}} \frac{\mathrm{d} z_{b}}{\mathrm{~d} Z} & =\left(\sum_{n=1, n \longrightarrow \text { odd }}^{\infty-1} n z_{b}^{n-1} e^{-i(n-1) \theta}+\sum_{n=2, n \longrightarrow \text { even }}^{\infty} n z_{b}^{n-1} e^{-i n \theta}\right) \frac{\mathrm{d} z_{b}}{\mathrm{~d} Z}, \\
\frac{\mathrm{d} Z_{B}}{\mathrm{~d} Z} & =\left(\frac{1}{z_{b}} \sum_{n=1, n \longrightarrow \text { odd }}^{\infty-1} n z_{b}^{n} e^{-i(n-1) \theta}+\frac{1}{z_{b}} \sum_{n=2, n \longrightarrow \text { even }}^{\infty} n z_{b}^{n} e^{-i n \theta}\right) \frac{\mathrm{d} z_{b}}{\mathrm{~d} Z}, \\
\frac{\mathrm{d} Z_{B}}{\mathrm{~d} Z} & =\left(\frac{1}{z_{b}}\left(z_{b}\left(\frac{1}{1-\left|z_{b}\right|^{2}}+\frac{2\left|z_{b}\right|^{2}}{\left(1-\left|z_{b}\right|^{2}\right)^{2}}\right)\right)+\frac{1}{z_{b}}\left(\frac{2\left|z_{b}\right|^{2}}{1-\left|z_{b}\right|^{2}}+\frac{2\left|z_{b}\right|^{4}}{\left(1-\left|z_{b}\right|^{2}\right)^{2}}\right)\right) \frac{\mathrm{d} z_{b}}{\mathrm{~d} Z} .
\end{aligned}
$$

This is simplified as follows:

$$
\begin{aligned}
& \frac{\mathrm{d} Z_{B}}{\mathrm{~d} Z}=\left(\left(\frac{1}{1-\left|z_{b}\right|^{2}}+\frac{2\left|z_{b}\right|^{2}}{\left(1-\left|z_{b}\right|^{2}\right)^{2}}\right)+\frac{1}{z_{b}}\left(\frac{2\left|z_{b}\right|^{2}}{1-\left|z_{b}\right|^{2}}+\frac{2\left|z_{b}\right|^{4}}{\left(1-\left|z_{b}\right|^{2}\right)^{2}}\right)\right) \frac{\mathrm{d} z_{b}}{\mathrm{~d} Z}, \\
& \frac{\mathrm{d} Z_{B}}{\mathrm{~d} Z}=\frac{1}{z_{b}}\left(\frac{\left(z_{b}+2\left|z_{b}\right|^{2}\right)}{\left(1-\left|z_{b}\right|^{2}\right)}+\frac{2\left(z_{b}\left|z_{b}\right|^{2}+\left|z_{b}\right|^{4}\right)}{\left(1-\left|z_{b}\right|^{2}\right)^{2}}\right) \frac{\mathrm{d} z_{b}}{\mathrm{~d} Z},
\end{aligned}
$$

where $\left(\mathrm{d} z_{b} / \mathrm{d} Z\right)$ and $z_{b}$ will be quantities lying inside the unit circle; therefore, the RHS is the equivalent first derivative for $Z_{B}$ in terms of the complex baselet $z_{b}$ of $Z_{B}$. Anything above first derivative is redundant in implementation because the second or higher derivatives can be implemented inside the unit circle with their own complex baselets. However, the complex conjugate $Z_{B}^{*}$ is not complex differentiable in the complex plane $Z$ except at $Z=0$ and also not holomorhic. $Z_{B}^{*}$ can also be implemented using the complex baselet $z_{b}$ of $Z_{B}$ as follows:

$$
Z_{B}^{*}=\sum_{n=1, n \longrightarrow \text { odd }}^{\infty-1} z_{b}^{n} e^{-i(n+1) \theta}+\sum_{n=2, n \longrightarrow \text { even }}^{\infty} z_{b}^{n} e^{-i n \theta} .
$$

This implementation is complex differentiable from Theorem 9, and also applying the chain rule,

$$
\begin{aligned}
\frac{\mathrm{d} Z_{B}^{*}}{\mathrm{~d} Z} & =\frac{\mathrm{d} Z_{B}^{*}}{\mathrm{~d} z_{b}} \frac{\mathrm{d} z_{b}}{\mathrm{~d} Z}=\left(\sum_{n=1, n \longrightarrow \text { odd }}^{\infty-1} n z_{b}^{n-1} e^{-i(n+1) \theta}+\sum_{n=2, n \longrightarrow \text { even }}^{\infty} n z_{b}^{n-1} e^{-i n \theta}\right) \frac{\mathrm{d} z_{b}}{\mathrm{~d} Z}, \\
\frac{\mathrm{d} Z_{B}^{*}}{\mathrm{~d} Z} & =\left(\frac{1}{z_{b}} \sum_{n=1, n \longrightarrow \text { odd }}^{\infty-1} n z_{b}^{n} e^{-i(n+1) \theta}+\frac{1}{z_{b}} \sum_{n=2, n \longrightarrow \text { even }}^{\infty} n z_{b}^{n} e^{-i n \theta}\right) \frac{\mathrm{d} z_{b}}{\mathrm{~d} Z}, \\
\frac{\mathrm{d} Z_{B}^{*}}{\mathrm{~d} Z} & =\left(\frac{1}{z_{b}}\left(z_{b}^{*}\left(\frac{1}{1-\left|z_{b}\right|^{2}}+\frac{2\left|z_{b}\right|^{2}}{\left(1-\left|z_{b}\right|^{2}\right)^{2}}\right)\right)+\frac{1}{z_{b}}\left(\left(\frac{2\left|z_{b}\right|^{2}}{1-\left|z_{b}\right|^{2}}+\frac{2\left|z_{b}\right|^{4}}{\left(1-\left|z_{b}\right|^{2}\right)^{2}}\right)\right)\right) \frac{\mathrm{d} z_{b}}{\mathrm{~d} Z},
\end{aligned}
$$

which can be simplified to the following expression:

$$
\begin{aligned}
& \frac{\mathrm{d} Z_{B}^{*}}{\mathrm{~d} Z}=\left(\frac{\left|z_{b}\right|^{2}}{z_{b}^{2}}\left(\frac{1}{1-\left|z_{b}\right|^{2}}+\frac{2\left|z_{b}\right|^{2}}{\left(1-\left|z_{b}\right|^{2}\right)^{2}}\right)+\frac{1}{z_{b}}\left(\left(\frac{2\left|z_{b}\right|^{2}}{1-\left|z_{b}\right|^{2}}+\frac{2\left|z_{b}\right|^{4}}{\left(1-\left|z_{b}\right|^{2}\right)^{2}}\right)\right)\right) \frac{\mathrm{d} z_{b}}{\mathrm{~d} Z}, \\
& \frac{\mathrm{d} Z_{B}^{*}}{\mathrm{~d} Z}=\frac{\left|z_{b}\right|^{2}}{z_{b}^{3}}\left(\frac{\left(z_{b}+2\left|z_{b}\right|^{2}\right)}{1-\left|z_{b}\right|^{2}}+\frac{2\left(z_{b}\left|z_{b}\right|^{2}+\left|z_{b}\right|^{4}\right)}{\left(1-\left|z_{b}\right|^{2}\right)^{2}}\right) \frac{\mathrm{d} z_{b}}{\mathrm{~d} Z} .
\end{aligned}
$$


Therefore, using the complex baselet-based implementation, $\left(\mathrm{d} Z_{B}^{*} / \mathrm{d} Z\right)$ exists for all $z_{b}$ except for $z_{b}=0 . Z_{B}^{*}$ which exists in $\mathbb{C}$ outside the unit circle does not satisfy Cauchy-Riemann equation and not analytic because it is not complex differentiable except at $Z=0$ is made complex differentiable using the complex baselet transform approach. It is infinitely differentiable and hence holomorphic in this implementation.

Theorem 11. The nonanalytic function $\left|Z_{B}\right|^{2}$ defined in $\mathbb{C}$, not satisfying the Cauchy-Riemann equations which constitute a necessary and sufficient condition for it to be complex differentiable in $\mathbb{C}$, can be made complex differentiable inside the unit circle using the complex baselet implementation and so any differential operation on $\left|Z_{B}\right|^{2}$ in $\mathbb{C}$, that does not exist, is made equivalent to a differential operation on the complex baselet-based implementation inside the unit circle, which always converges and hence exists.
Proof. The complex baselet implementation of $Z_{B}$ inside unit circle is as follows:

$$
Z_{B}=\sum_{n=1, n \longrightarrow \text { odd }}^{\infty-1} z_{b}^{n} e^{-i(n-1) \theta}+\sum_{n=2, n \longrightarrow \text { even }}^{\infty} z_{b}^{n} e^{-i n \theta}
$$

Furthermore, $\left|Z_{B}\right|^{2}=Z_{B} Z_{B}^{*}$ in $\mathbb{C}$, outside the unit circle,

$$
\frac{\mathrm{d}\left|Z_{B}\right|^{2}}{\mathrm{~d} Z}=\frac{\mathrm{d}\left|Z_{B}\right|^{2}}{\mathrm{~d} z_{b}} \frac{\mathrm{d} z_{b}}{\mathrm{~d} Z}
$$

However, $\left(\mathrm{d}\left|Z_{B}\right|^{2} / \mathrm{d} z_{b}\right)=\left(\mathrm{d}\left(Z_{B} Z_{B}^{*}\right) / \mathrm{d} z_{b}\right)$.

$$
\frac{\mathrm{d}\left|Z_{B}\right|^{2}}{\mathrm{~d} z_{b}}=Z_{B} \frac{\mathrm{d} Z_{B}^{*}}{\mathrm{~d} z_{b}}+Z_{B}^{*} \frac{\mathrm{d} Z_{B}}{\mathrm{~d} z_{b}} \text {. }
$$

From Theorem 10,

$$
\begin{aligned}
Z_{B}^{*} & =\sum_{n=1, n \longrightarrow \text { odd }}^{\infty-1} z_{b}^{n} e^{-i(n+1) \theta}+\sum_{n=2, n \longrightarrow \text { even }}^{\infty} z_{b}^{n} e^{-i n \theta}, \\
\frac{\mathrm{d} Z_{B}}{\mathrm{~d} z_{b}} & =\frac{1}{z_{b}}\left(\frac{\left(z_{b}+2\left|z_{b}\right|^{2}\right)}{\left(1-\left|z_{b}\right|^{2}\right)}+\frac{2\left(z_{b}\left|z_{b}\right|^{2}+\left|z_{b}\right|^{4}\right)}{\left(1-\left|z_{b}\right|^{2}\right)^{2}}\right) \frac{\mathrm{d} z_{b}}{\mathrm{~d} Z}, \\
\frac{\mathrm{d} Z_{B}^{*}}{\mathrm{~d} z_{b}} & =\frac{\left|z_{b}\right|^{2}}{z_{b}^{3}}\left(\frac{\left(z_{b}+2\left|z_{b}\right|^{2}\right)}{\left(1-\left|z_{b}\right|^{2}\right)}+\frac{2\left(z_{b}\left|z_{b}\right|^{2}+\left|z_{b}\right|^{4}\right)}{\left(1-\left|z_{b}\right|^{2}\right)^{2}}\right) \frac{\mathrm{d} z_{b}}{\mathrm{~d} Z} .
\end{aligned}
$$

Therefore,

$$
\begin{aligned}
\frac{\mathrm{d}\left|Z_{B}\right|^{2}}{\mathrm{~d} z_{b}}= & \left(\sum_{n=1, n \longrightarrow \mathrm{odd}}^{\infty-1} z_{b}^{n} e^{-i(n-1) \theta}+\sum_{n=2, n}^{\infty} z_{b}^{n} e^{-i n \theta}\right)\left(\frac{\left|z_{b}\right|^{2}}{z_{b}^{3}}\left(\frac{\left(z_{b}+2\left|z_{b}\right|^{2}\right)}{\left(1-\left|z_{b}\right|^{2}\right)}+\frac{2\left(z_{b}\left|z_{b}\right|^{2}+\left|z_{b}\right|^{4}\right)}{\left(1-\left|z_{b}\right|^{2}\right)^{2}}\right)\right) \frac{\mathrm{d} z_{b}}{\mathrm{~d} Z}+ \\
& \left(\left(\sum_{n=1, n \longrightarrow \text { odd }}^{\infty-1} z_{b}^{n} e^{-i(n+1) \theta}+\sum_{n=2, n \longrightarrow \text { even }}^{\infty} z_{b}^{n} e^{-i n \theta}\right) \frac{1}{z_{b}}\left(\frac{\left(z_{b}+2\left|z_{b}\right|^{2}\right)}{\left(1-\left|z_{b}\right|^{2}\right)}+\frac{2\left(z_{b}\left|z_{b}\right|^{2}+\left|z_{b}\right|^{4}\right)}{\left(1-\left|z_{b}\right|^{2}\right)^{2}}\right)\right)\left(\frac{\mathrm{d} z_{b}}{\mathrm{~d} Z}\right) .
\end{aligned}
$$


Then, the following relation is obtained:

$$
\begin{aligned}
& \frac{\mathrm{d}\left|Z_{B}\right|^{2}}{\mathrm{~d} Z}=\left(\begin{array}{l}
\left(\sum_{n=1, n \longrightarrow \text { odd }}^{\infty-1} z_{b}^{n} e^{-i(n-1) \theta}+\sum_{n=2, n \longrightarrow \text { even }}^{\infty} z_{b}^{n} e^{-i n \theta}\right)\left(\frac{\left|z_{b}\right|^{2}}{z_{b}^{3}}\left(\frac{\left(z_{b}+2\left|z_{b}\right|^{2}\right)}{\left(1-\left|z_{b}\right|^{2}\right)}+\frac{2\left(z_{b}\left|z_{b}\right|^{2}+\left|z_{b}\right|^{4}\right)}{\left(1-\left|z_{b}\right|^{2}\right)^{2}}\right)\right)+ \\
\left.\left(\sum_{n=1, n \longrightarrow \text { odd }}^{\infty-1} z_{b}^{n} e^{-i(n+1) \theta}+\sum_{n=2, n \longrightarrow \text { even }}^{\infty} z_{b}^{n} e^{-i n \theta}\right) \frac{1}{z_{b}}\left(\frac{\left(z_{b}+2\left|z_{b}\right|^{2}\right)}{\left(1-\left|z_{b}\right|^{2}\right)}+\frac{2\left(z_{b}\left|z_{b}\right|^{2}+\left|z_{b}\right|^{4}\right)}{\left(1-\left|z_{b}\right|^{2}\right)^{2}}\right)\right)
\end{array}\right) \\
& \left.\frac{\mathrm{d}\left|Z_{B}\right|^{2}}{\mathrm{~d} Z}=\left(\frac{\left(z_{b}+2\left|z_{b}\right|^{2}\right)}{\left(1-\left|z_{b}\right|^{2}\right)}+\frac{2\left(z_{b}\left|z_{b}\right|^{2}+\left|z_{b}\right|^{4}\right)}{\left(1-\left|z_{b}\right|^{2}\right)^{2}}\right)\left(\begin{array}{c}
\left(\frac{\left|z_{b}^{2}\right|}{z_{b}^{3}}\right)\left(\sum_{n=1, n \rightarrow \text { odd }}^{\infty-1} z_{b}^{n} e^{-i(n-1) \theta}+\sum_{n=2, n \rightarrow \text { even }}^{\infty} z_{b}^{n} e^{-i n \theta}\right)+ \\
\frac{1}{z_{b}}\left(\sum_{n=1, n \longrightarrow \text { odd }}^{\infty-1} z_{b}^{n} e^{-i(n+1) \theta}+\sum_{n=2, n \longrightarrow \text { even }}^{\infty} z_{b}^{n} e^{-i n \theta}\right)
\end{array}\right)\right) \frac{\mathrm{d} z_{b}}{\mathrm{~d} Z},
\end{aligned}
$$

which exists and always converges. Thus, using the complex baselet implementation, $\left(\mathrm{d}\left|Z_{B}\right|^{2} / \mathrm{d} Z\right)$ exists for all $z_{b}$ except for $z_{b}=0$.

$\left|Z_{B}\right|^{2}$, which exists in $\mathbb{C}$ outside the unit circle does not satisfy Cauchy-Riemann equation and not holomorphic because it is not complex differentiable, is made complex differentiable using the complex baselet transform approach. It is infinitely differentiable and hence holomorphic in this implementation.

Theorem 12. The nonanalytic function $\left|Z_{B}\right|$ defined in $\mathbb{C}$, not satisfying the Cauchy-Riemann equations which constitute a necessary and sufficient condition for it to be complex differentiable in $\mathbb{C}$, can be made complex differentiable inside the unit circle using the complex baselet implementation. Thus, any differential operation on $\left|Z_{B}\right|$ in $\mathbb{C}$, that does not exist, is made equivalent to a differential operation on the complex baselet-based implementation inside the unit circle, which always converges and therefore exists.

Proof. The complex baselet implementation of $Z_{B}$ inside unit circle is as follows:

$$
Z_{B}=\sum_{n=1, n \longrightarrow \text { odd }}^{\infty-1} z_{b}^{n} e^{-i(n-1) \theta}+\sum_{n=2, n \longrightarrow \text { even }}^{\infty} z_{b}^{n} e^{-i n \theta} .
$$

Furthermore, $\left|Z_{B}\right|=\left(Z_{B} Z_{B}^{*}\right)^{(1 / 2)}=Z_{B}^{(1 / 2)} Z_{B}^{*(1 / 2)}$ in $\mathbb{C}$, outside the unit circle,

$$
\frac{\mathrm{d}\left|Z_{B}\right|}{\mathrm{d} Z}=\frac{\mathrm{d}\left|Z_{B}\right|}{\mathrm{d} z_{b}} \frac{\mathrm{d} z_{b}}{\mathrm{~d} Z}
$$

However, $\left(\mathrm{d}\left|Z_{B}\right| / \mathrm{d} z_{b}\right)=\left(\mathrm{d}\left(Z_{B}^{(1 / 2)} Z_{B}^{*(1 / 2)}\right) / \mathrm{d} z_{b}\right)$.

$$
\begin{aligned}
& \frac{\mathrm{d}\left|Z_{B}\right|}{\mathrm{d} z_{b}}=Z_{B}^{(1 / 2)} \frac{\mathrm{d} Z_{B}^{*(1 / 2)}}{\mathrm{d} z_{b}}+Z_{B}^{*(1 / 2)} \frac{\mathrm{d} Z_{B}^{(1 / 2)}}{\mathrm{d} z_{b}} \\
& Z_{B}^{(1 / 2)}=\left(\sum_{n=1, n \longrightarrow \text { odd }}^{\infty-1} z_{b}^{n} e^{-i(n-1) \theta}+\sum_{n=2, n \longrightarrow \text { even }}^{\infty} z_{b}^{n} e^{-i n \theta}\right)^{(1 / 2)} .
\end{aligned}
$$

Equation (66) is converging.

$$
\begin{aligned}
\frac{\mathrm{d} Z_{B}^{(1 / 2)}}{\mathrm{d} z_{b}} & =\frac{1}{2\left(\sum_{n=1, n \longrightarrow \mathrm{odd}}^{\infty-1} z_{b}^{n} e^{-i(n-1) \theta}+\sum_{n=2, n \longrightarrow \mathrm{even}}^{\infty} z_{b}^{n} e^{-i n \theta}\right)^{(1 / 2)}} \frac{\mathrm{d} Z_{B}}{\mathrm{~d} z_{b}} \\
& =\frac{1}{2\left(\sum_{n=1, n \longrightarrow \mathrm{odd}}^{\infty-1} z_{b}^{n} e^{-i(n-1) \theta}+\sum_{n=2, n \longrightarrow \mathrm{even}}^{\infty} z_{b}^{n} e^{-i n \theta}\right)^{(1 / 2)}} \frac{1}{z_{b}}\left(\frac{\left(z_{b}+2\left|z_{b}\right|^{2}\right)}{\left(1-\left|z_{b}\right|^{2}\right)}+\frac{2\left(z_{b}\left|z_{b}\right|^{2}+\left|z_{b}\right|^{4}\right)}{\left(1-\left|z_{b}\right|^{2}\right)^{2}}\right) \frac{\mathrm{d} z_{b}}{\mathrm{~d} Z} \\
Z_{B}^{*(1 / 2)} & =\left(\sum_{n=1, n \longrightarrow \text { odd }}^{\infty-1} z_{b}^{n} e^{-i(n+1) \theta}+\sum_{n=2, n}^{\infty} z_{b}^{n} e^{-i n \theta}\right)^{(1 / 2)}
\end{aligned}
$$


Equation (68) is converging.

$$
\begin{aligned}
& \frac{\mathrm{d} Z_{B}^{*(1 / 2)}}{\mathrm{d} z_{b}}=\frac{1}{2\left(\sum_{n=1, n \longrightarrow \mathrm{dd}}^{\infty-1} z_{b}^{n} e^{-i(n+1) \theta}+\sum_{n=2, n \longrightarrow \mathrm{even}}^{\infty} z_{b}^{n} e^{-i n \theta}\right)^{(1 / 2)}} \frac{\mathrm{d} Z_{B}^{*}}{\mathrm{~d} z_{b}}, \\
& \frac{\mathrm{d} Z_{B}^{*(1 / 2)}}{\mathrm{d} z_{b}}=\frac{1}{2\left(\sum_{n=1, n \longrightarrow \mathrm{odd}}^{\infty-1} z_{b}^{n} e^{-i(n+1) \theta}+\sum_{n=2, n \longrightarrow \mathrm{even}}^{\infty} z_{b}^{n} e^{-i n \theta}\right)^{(1 / 2)}} \frac{\left|z_{b}\right|^{2}}{z_{b}^{3}}\left(\frac{\left(z_{b}+2\left|z_{b}\right|^{2}\right)}{\left(1-\left|z_{b}\right|^{2}\right)}+\frac{2\left(z_{b}\left|z_{b}\right|^{2}+\left|z_{b}\right|^{4}\right)}{\left(1-\left|z_{b}\right|^{2}\right)^{2}}\right) \frac{\mathrm{d} z_{b}}{\mathrm{~d} Z}
\end{aligned}
$$

Substituting equations (66), (67), (68), and (69) in equation (65),

$$
\begin{aligned}
\frac{\mathrm{d}\left|Z_{B}\right|}{\mathrm{d} z_{b}}= & \frac{\left|z_{b}\right|^{2}}{z_{b}^{3}} \frac{\left(\sum_{n=1, n \longrightarrow \mathrm{odd}}^{\infty-1} z_{b}^{n} e^{-i(n-1) \theta}+\sum_{n=2, n \longrightarrow \mathrm{even}}^{\infty} z_{b}^{n} e^{-i n \theta}\right)^{(1 / 2)}}{2\left(\sum_{n=1, n \longrightarrow \mathrm{odd}}^{\infty-1} z_{b}^{n} e^{-i(n+1) \theta}+\sum_{n=2, n \longrightarrow \text { even }}^{\infty} z_{b}^{n} e^{-i n \theta}\right)^{(1 / 2)}}\left(\frac{\left(z_{b}+2\left|z_{b}\right|^{2}\right)}{\left(1-\left|z_{b}\right|^{2}\right)^{2}}+\frac{2\left(z_{b}\left|z_{b}\right|^{2}+\left|z_{b}\right|^{4}\right)}{\left(1-\left|z_{b}\right|^{2}\right)^{2}}\right) \frac{\mathrm{d} z_{b}}{\mathrm{~d} Z}+ \\
& \frac{\left(\sum_{n=1, n \longrightarrow \mathrm{odd}}^{\infty-1} z_{b}^{n} e^{-i(n+1) \theta}+\sum_{\left.n=2, n \longrightarrow \text { even } z_{b}^{n} e^{-i n \theta}\right)^{(1 / 2)}}^{\infty}\right.}{2 z_{b}\left(\sum_{n=1, n \longrightarrow \mathrm{odd}}^{\infty-1} z_{b}^{n} e^{-i(n-1) \theta}+\sum_{n=2, n \longrightarrow \text { even }}^{\infty} z_{b}^{n} e^{-i n \theta}\right)^{(1 / 2)}}\left(\frac{\left(z_{b}+2\left|z_{b}\right|^{2}\right)}{\left(1-\left|z_{b}\right|^{2}\right)}+\frac{2\left(z_{b}\left|z_{b}\right|^{2}+\left|z_{b}\right|^{4}\right)}{\left(1-\left|z_{b}\right|^{2}\right)^{2}}\right) \frac{\mathrm{d} z_{b}}{\mathrm{~d} Z}
\end{aligned}
$$

Therefore,

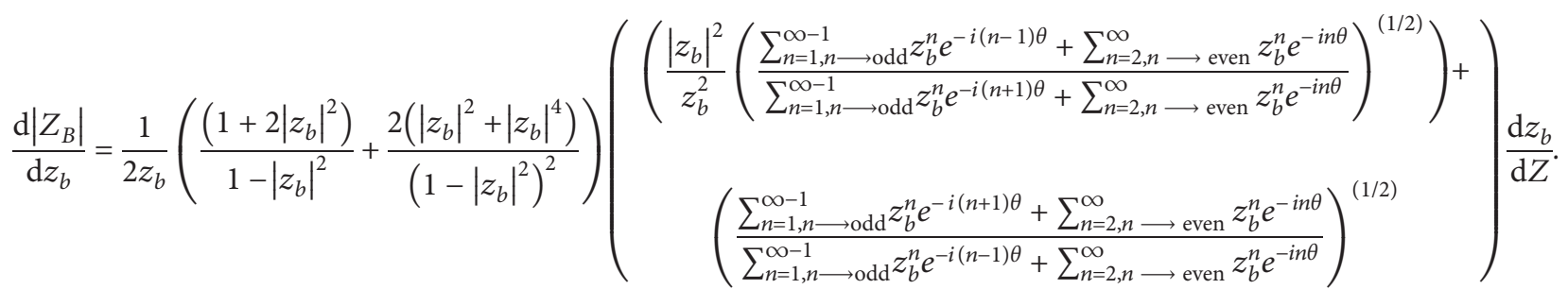

This exists and always converges. Consequently, (d| $\left.Z_{B} \mid / d Z\right)$ exists for all $z_{b}$ except for $z_{b}=0$.

Therefore, $\left|Z_{B}\right|$ that exists in $\mathbb{C}$ outside the unit circle and not satisfying the Cauchy-Riemann equations because it is not complex differentiable is made complex differentiable using the complex baselet transform approach. The transformed expression is infinitely differentiable and hence holomorphic in this implementation.

\section{Implementation Unit of Complex Baselet Transform}

The implementation unit of $Z_{B}$ using its complex baselet, $z_{b}$, is displayed in Figure 1. This structure follows directly from Part 1 of the proof of Theorem 1. A toggle switch with initial position at 1 and toggle frequency $f=\left(1 / t_{\text {mult }}\right)$, where $t_{\text {mult }}$ is the time of computation of the multiplier, forms the first input of the multiplier. The second input constitutes a feedback loop of the multiplier output as shown, with the initial condition for this input being unity. The accumulator unit consecutively sums the components of the progressive series. The series being infinite but converging in nature demands only a finite and limited memory storage of the accumulator, that is, designed to have a settling time $T_{s}$, after which only progressive series is made available at the output. This settling time allows sufficient number of terms being summed up in the infinite series so that the output will be the complex baselet transform implementation of $Z_{B}$, from its complex baselet input $z_{b}$, with the designed accuracy. The symbolic block representation of complex baselet transform is displayed in Figure 2. 


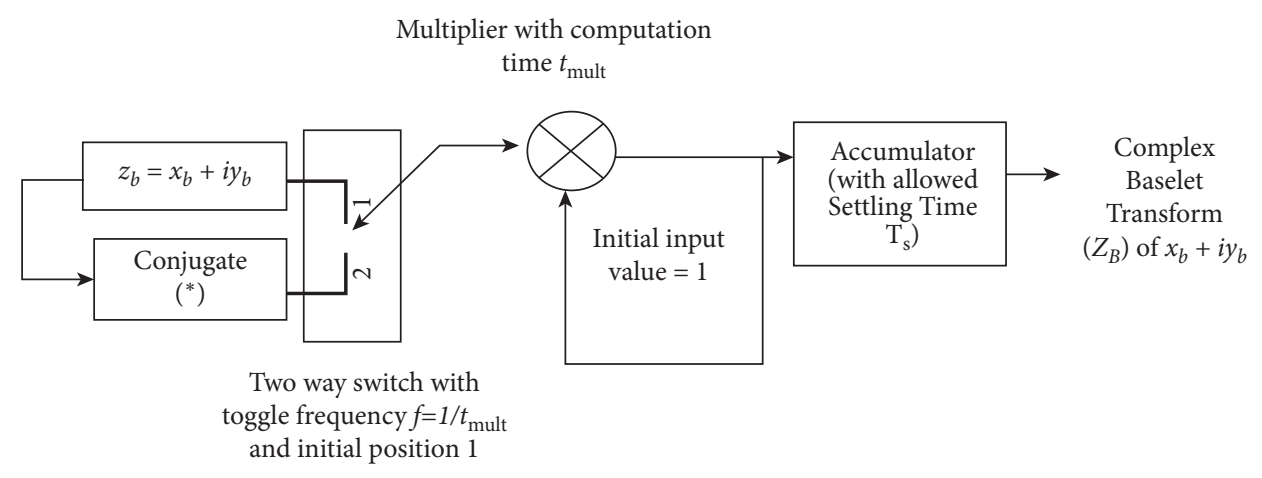

FIgURE 1: Implementation unit of $\mathrm{Z}_{B}$ using complex baselet.

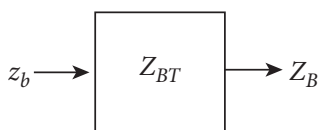

FIgURE 2: Symbolic block representation of complex baselet transform.

\section{Conclusions}

Stability along with holomorphicity constitutes a significant and desirable property set for many complex functions in $\mathbb{C}$ plane. A transform-based implementation using complex baselet transform is proposed that comprises an infinite converging series of progressive products of a base unit called complex baselet and its conjugate only. Various operations applied on the original complex function are mapped to equivalent operations on the complex baselet inside the unit circle. This method of implementation has got a distinct feature that the component elements are all lying inside the unit circle. Theorems and lemmas related to the proposed approach are also presented with proofs. A stable implementation of a normally unstable complex function is developed using the approach. Additionally, functions not satisfying Cauchy-Riemann equations and consequently not holomorphic in $\mathbb{C}$ are made complex differentiable with the proposed transform-based implementation. Analysis can be extended further for the proposed approach, which can reveal the in-depth geometrical interpretation and patterns.

\section{Data Availability}

The data used to support the findings of this study are included within the article.

\section{Conflicts of Interest}

The author declares that there are no conflicts of interest.

\section{Acknowledgments}

The author gratefully acknowledges the support provided by the Department of Applied Sciences, Govt. Model Engineering College-APJ Abdul Kalam Technological University, Ernakulam, India, for carrying out this research work. The author would also like to thank Editage (http://www.editage. com) for the English language editing services.

\section{References}

[1] L. Ahlfors, Complex Analysis, McGraw-Hill, New York, NY, USA, 3rd edition, 1979.

[2] A. S. Kucik, "Carleson measures for hilbert spaces of analytic functions on the complex half-plane," Journal of Mathematical Analysis and Applications, vol. 445, no. 1, pp. 476-497, 2017.

[3] T. Binesh, A Transform Based Infinite Series Implementation Inside Unit Circle for Any Complex Number or Function and its Applications, https://papers.ssrn.com/sol3/papers.cfm? abstract_id=3512147, 2019.

[4] E. T. Whittaker and G. N. Watso, A Course of Modern Analysis, Cambridge University Press, Cambridge, England, 1979.

[5] R. Spiegel Murray, Theory and Problems of Complex Variables-with an Introduction to Conformal Mapping and its Applications, McGraw-Hill, New York, NY, USA, 3rd edition, 1964.

[6] S. Bochner and K. Chandrasekkharan, "Fourier transform," Annals of Mathematics Studies, vol. 19, p. 3, 1949.

[7] T. Ohsawa, "Completeness of noncompact analytic spaces," Publications of the Research Institute for Mathematical Sciences, vol. 20, no. 3, pp. 683-692, 1984.

[8] M. Mittal and V. I. Paulsen, "Operator algebras of functions," Journal of Functional Analysis, vol. 258, no. 9, pp. 3195-3225, 2010.

[9] S. N. Mergelyan, "Uniform approximation to functions of a complex variables," Uspekhi Mathematical Nauk (Russia Math. Surveys), vol. 7, no. 2, pp. 31-122, 1952.

[10] F. K. Yu, "Uniform n-analytic polynomial approximations of functions on rectifiable contours in C," Mathematical Notes, vol. 59, no. 4, pp. 435-439, 1996.

[11] A. Boivin, P. M. Gauthier, and P. V. Paramonov, "Uniform approximation on closed subsets of $\mathrm{C}$ by polyanalytic functions," Izvestiya: Mathematics, vol. 68, no. 3, pp. 447-459, 2004.

[12] R. Narasimhan, Analysis on Real and Complex Manifolds(Advanced Studies in Pure Mathematics), North-Holland publication, Amsterdam, Netherlands, 1st edition, 1964.

[13] J. Verdera, " $C^{m}$-approximation by solution of elliptic equations and calderon-zygmund operators," Duke Mathematical Journal, vol. 55, no. 1, pp. 157-187, 1987.

[14] A. Aziz, "Inequalities for the polar derivative of a polynomial," Journal of Approximation Theory, vol. 55, no. 2, pp. 183-193, 1988. 
[15] E. M. Stein and G. Weiss, "Generalization of the cauchyriemann equations and representations of the rotation group," American Journal of Mathematics, vol. 90, no. 1, pp. 163-196, 1968.

[16] A. Grinshpan, D. S. Kaliuzhnyi-Verbovetskyi, V. Vinnikov, and H. J. Woerdeman, "Stable and real-zero polynomials in two variables," Multidimensional Systems and Signal Processing, vol. 27, no. 1, pp. 1-26, 2016.

[17] B. J. Levin, "Distribution of zeros of entire functions," in Translations of Mathematical Monographs, vol. 5, Revised Edition, Translated from Russian by R. P. Boas, J. M. Danskin, F. M. Goodspeed, J. Korevaar, A. L. Shields and H. P. Thielman, American Mathematical Society, Providence, RI, USA, 1980.

[18] C. G. Gal, S. G. Gal, and J. A. Goldstein, "Evolution Equations with real time variable and complex spatial variables," Complex Variables and Elliptic Equations, vol. 53, no. 8, pp. 753-774, 2014.

[19] M. E. Van Valkenburg, Network Analysis, Prentice Hall Inc, Englewood Cliffs, NJ, USA, 2st edition, 1964.

[20] L. D. Landau and E. M. Lifshitz, Quantum Mechanics: Non Relativistic Theory, Butterworth-Heinemann, Oxford, UK, 3rd edition, 1991.

[21] F. Strocchi, "Complex coordinates and quantum mechanics," Reviews of Modern Physics, vol. 38, no. 1, pp. 36-40, 1966.

[22] L. Faddeev, A. Slavnov, and G. Fields, Introduction to Quantum Theory, Benjamin Cummings, San Francisco, CA, USA, 1980.

[23] M. Blasone and L. Smaldone, "Functional integrals and inequivalent representations in quantum field theory," Annals of Physics, vol. 383, pp. 207-238, 2017. 